\title{
Long-Term ECG Trends in Atherosclerotic Mouse Subjects
}

\author{
MB Oefinger, M Krieger, RG Mark \\ Massachusetts Institute of Technology, Cambridge, MA, USA
}

\begin{abstract}
The double-knockout (dKO) mouse model, with homozygous null encoding for the apoE lipoprotein molecule and SRB-I receptor, shows extremely elevated LDL and severely depressed HDL levels in blood serum. The subjects show 100\% mortality by the age of 8 weeks, with accompanying cardiac hypertrophy, reduced ejection fraction and high incidence of atherosclerosis and multiple regions of myocardial infarct noted on necropsy. While these gross observations have been published previously, we now present long-term ECG trends of these subjects. Of specific note in the dKO subjects are the appearance of severe bradycardia during the night and early morning; high-amplitude ultradian rhythm fluctuations relative to wild-type mice; STsegment elevation and depression; and an array of ECG anomalies ranging from bigeminy to 2 nd and 3rd degree heart block. While the dKO mice can, and periodically do, experience self-limited episodes of ventricular tachycardia and subsequent fibrillation, the small size of the heart makes sustained re-entry and fibrillation impossible (in accordance with the critical mass hypothesis). Our study of $11 \mathrm{dKO}$ subjects indicates that these subjects are not suffering a tachyarrhythmic death, but rather a progressive bradyarrhythmia and terminal asystolic cardiac arrest.
\end{abstract}

\section{Introduction}

MIT's Krieger Lab (Department of Biology) developed a genotype with homozygous null encoding for the apoE lipoprotein, a critical component in modulating levels of LDL ("bad cholesterol"), and SRB-I receptors, involved in biochemical pathways associated with HDL ("good cholesterol"). The phenotypic result of this "doubleknockout" (dKO) model is elevated serum LDL and reduced serum HDL [1]. Prior to studies of the dKO murine genotype, investigators had studied a singleknockout (KO) genotype in which the subjects had homozygous null encoding for apoE. While the KO genotype had extremely elevated serum LDL, it did not exhibit the infarcts, hypertrophy, reduced ejection fraction and shortened lifespan noted in the dKO genotype.
The focus of this study was the disease progression of dKO subjects as manifested in long-term ECG trends. To this end we measured ECG continuously in $11 \mathrm{dKO}$ subjects and compared the long-term ECG characteristics with wild-type (control) subjects. We reviewed heart rate, time-domain heart rate variability, arrhythmia and STsegments in the mice to ascertain a "typical" disease trajectory in $\mathrm{dKO}$ mice.

\section{Methods}

Each subject was anesthetized with isoflurane gas and three electrodes were implanted subcutaneously in a leadII equivalent configuration: the two electrodes in the thorax comprised the lead axis while the third electrode, implanted dorsally, served as an active ground electrode. Each subject was implanted at the age of approximately 31 days after weaning from the mother.

For data collection we used the Hermes (TM) preclinical physiological data collection and analysis system designed by Oefinger et. al. [2,3]. Using this system we collected ECG data continuously at $2 \mathrm{kHZ}$ for the lifespan of the subject (2-8 weeks for dKOs). At the end of the subject's life we ended recording and studied the heart rate trend plots generated automatically by the Hermes system, using the provided interactive graphical utility to focus on areas of interest in the ECG. In so doing we found areas of arrhythmia and abnormal heart rate variability in $\mathrm{dKO}$ subjects. Correlated with extrema in heart rate we noted ST-segment deviations, the details of which are discussed further in the Results section.

\section{Results}

Figure 1 shows a sample comparison of long-term heart rate in a control and $\mathrm{dKO}$ subject. Careful review of multiple records revealed the extreme dips in heart rate in $\mathrm{dKO}$ mice are most often sinus bradycardia in the earlier days of recording, while in later days the extreme dips are frequently associated with 2nd or 3rd degree heart block. Of additional note in the comparison of long-term heart rate is the very high amplitude ultradian rhythm fluctuation - fluctuations in heart rate that occur with periodicity of less than 24 hours - in the dKO subject. 

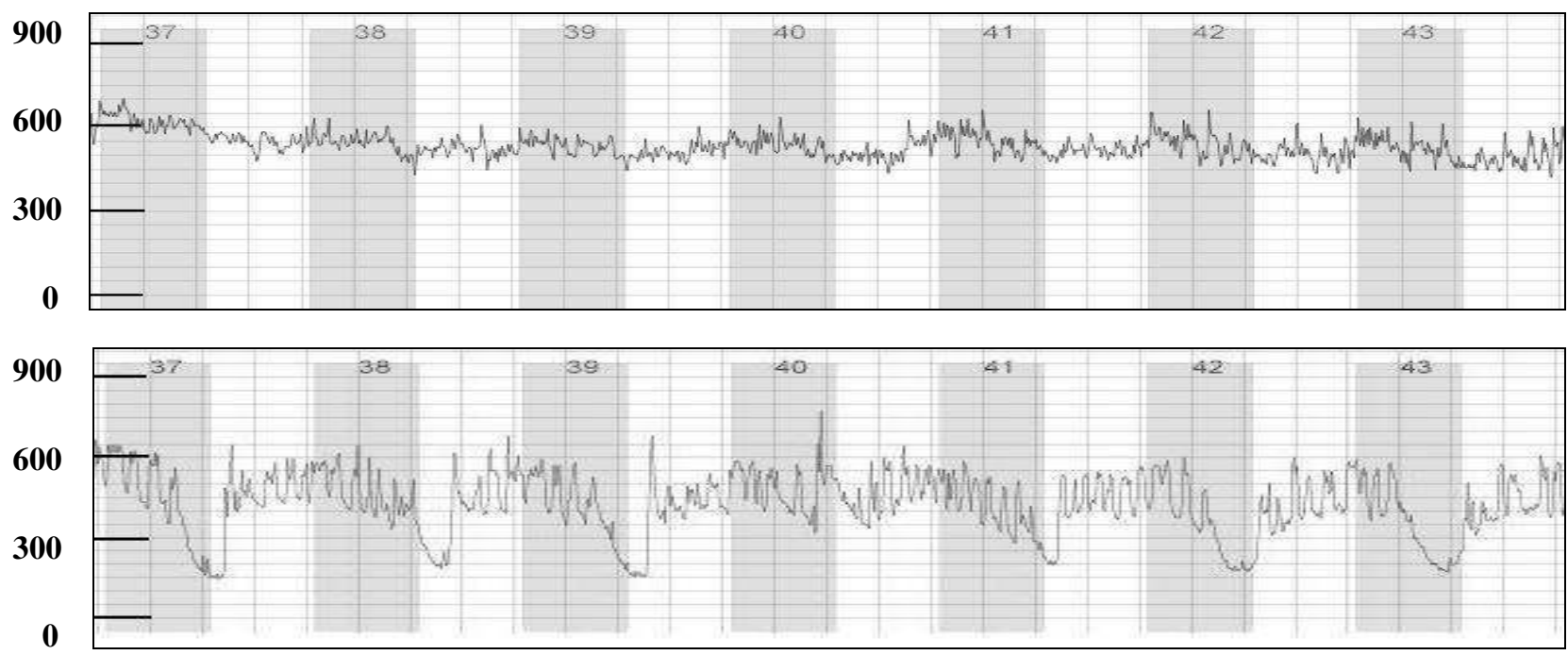

Figure 1: A comparison of wild-type (top) and dKO (bottom) long-term heart rate trends. The $x$-axis number represents the age of the subject (in days) and the y-axis number represents HR (in bpm). Light and dark cycles are shaded..

A closer examination of the ECG during heart rate extrema in the dKO shows frequent ST-segment elevation occurring during bradycardic episodes and ST-segment depression during normal sinus rhythm. While we only present one example below (where the heart rate plot is a zoomed version of figure 1 above) to illustrate such ST-segment changes in a single subject, the ST-segment elevation during bradycardia and STsegment depression during normal sinus rhythm was typical of the dKO subjects studied.

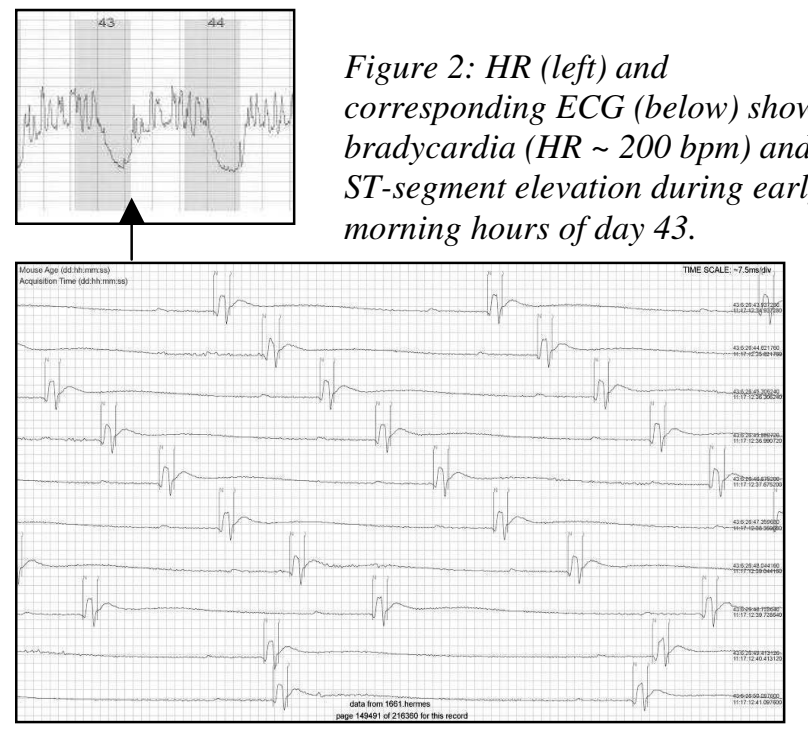

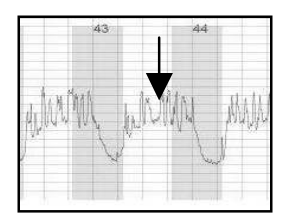

Figure 3: HR (left) and corresponding ECG (below) show sinus rhythm (HR $\sim 600 \mathrm{bpm})$ and $S T$-segment depression during late afternoon hours of day 43.

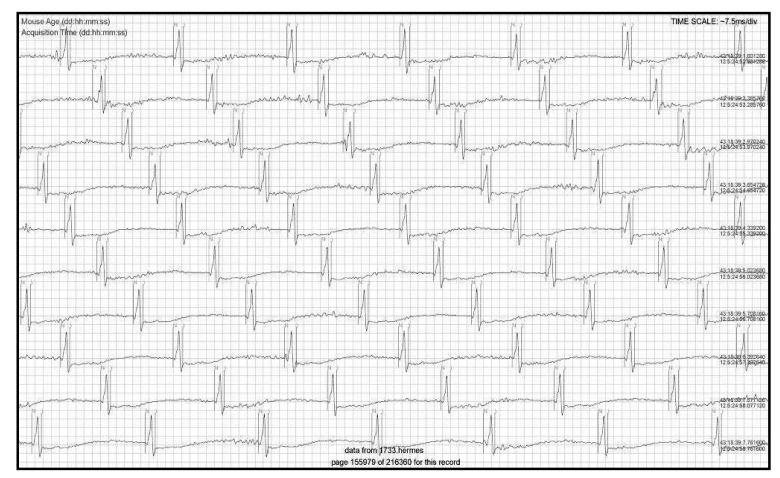

Review of other HR dips for the particular subject illustrated in figures 1,2 and 3 above reveals similar episodes of bradycardia and accompanying ST-segment elevation with ST-segment depression occurring upon resumption of recovery from bradycardia. A review of the ECG during the last minutes of life for this subject is shown below, illustrating a substantial ST-segment elevation episode (probable MI), 2:1 heart block, ectopic beats, and slowly widening QRS (probable intraventricular conduction defect). The heart rate 
continues to decrease and the QRS complex continues to widen until the subject becomes asystolic.

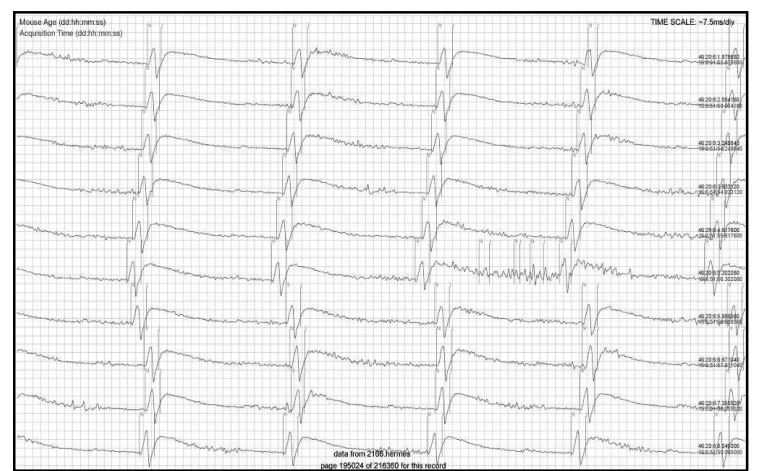

Figure 4: At 12 minutes before asystolic cardiac arrest, the ECG shows a massive ST-segment elevation, indicating probable MI.

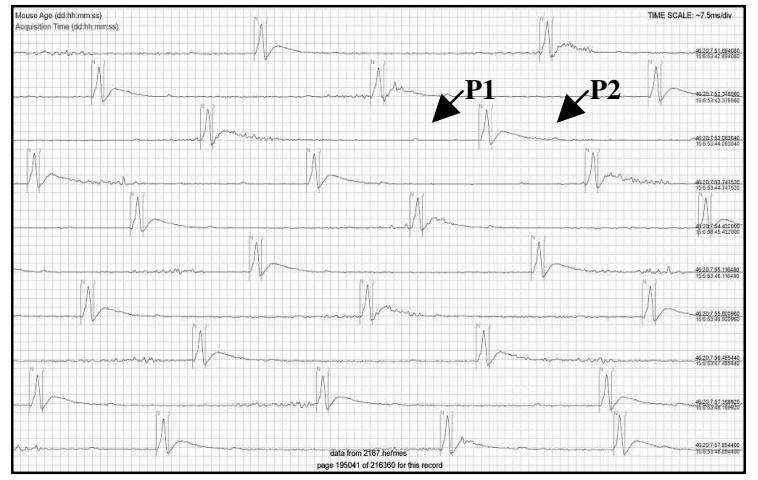

Figure 5: At approximately 10 minutes before asystole, the ECG shows 2:1 heart block. Label PI highlights a conducted P-wave and label P2 highlights a non-conducted $P$-wave.

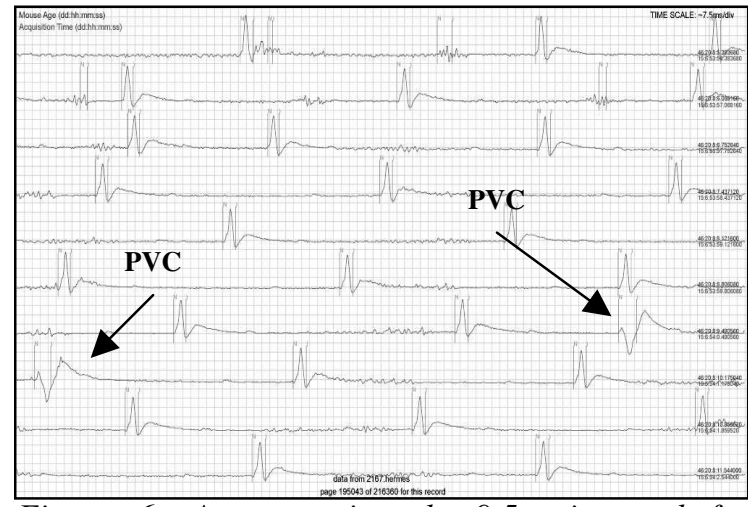

Figure 6: At approximately 9.5 minutes before asystole the ECG shows several PVCs.

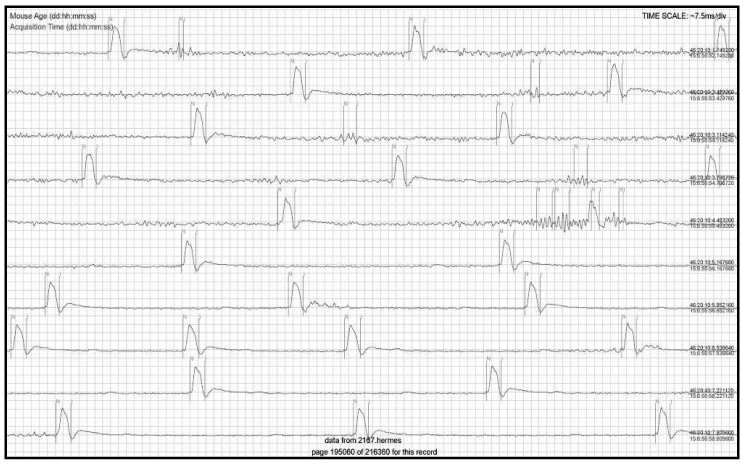

Figure 7: At 8 minutes before asystole the QRS complex has become substantially wider, indicating slowing of intraventricular conduction velocity.

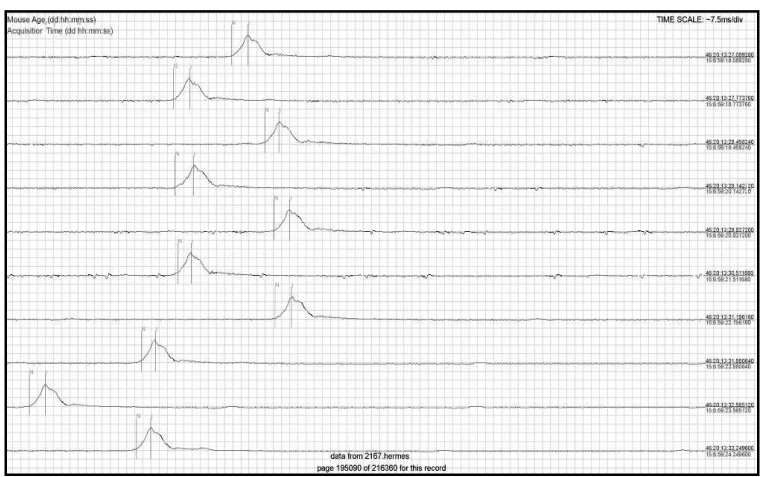

Figure 8: At 5 minutes before asystole the heart rate has slowed to approximately $60 \mathrm{bpm}$ and the QRS complex continues to widen. The trend of QRS widening and progressive bradycardia continues until asystole.

\section{Discussion and conclusions}

While the above results section details one particular dKO subject's disease course as manifested by ECG, a similar pattern of severe bradycardia $(\sim 200 \mathrm{bpm})$ in the late night and early morning hours appears in all 11 dKO subjects we studied. The association of bradycardia with ST-segment elevation and normal sinus rhythm ( 500-700 bpm) with ST-segment depression was a common finding in most, but not all, dKO records. Nearly all 11 dKO records showed extreme time-domain ultradian heart rate fluctuations with periodicity of about 1-3 hours and deviation of about 150-200 bpm.

The mode of death in $\mathrm{dKO}$ mice, as evidenced from our study of 11 subjects, appears to be progressive bradyarrhythmia and terminal asystolic cardiac arrest. 


\section{Acknowledgements}

This research was supported by NIH grant PO1HL66105.

\section{References}

[1] Krieger, M. (April 1998) Proc. Natl. Acad. Sci. USA, vol. 954077

[2] Oefinger, M et al. An Interactive Web-based Tool for Multi-Scale Physiological Data Visualization. Computers in Cadiology 2004

[3] Oefinger, M et al. System for Remote Multi-Channel Real-Time Monitoring of ECG via the Internet. Computers in Cardiology 2004
Address for correspondence

Matt Oefinger

Massachusetts Institute of Technology

77 Massachusetts Avenue, E25-505

Cambridge, MA 02139

oefinger@mit.edu 